\title{
An Unusual Case of Non-Reentrant Atrioventricular Nodal Tachycardia
}

\author{
Craig W. Raphael ${ }^{1}$ and Behzad Pavri ${ }^{1}$ \\ ${ }^{1}$ Thomas Jefferson University
}

January 3, 2022

\begin{abstract}
Although uncommonly encountered, dual atrioventricular nodal non-reentrant tachycardia (DAVNNRT) is a well described arrhythmia that can manifest in patients with dual atrioventricular nodal pathways physiology. This arrhythmia is characterized on ECG by a single P wave followed by two conducted QRS complexes, and on intracardiac EGMs by a single atrial electrogram followed by two separate His deflections and ventricular electrograms. Our case demonstrates a rare case of "triple atrioventricular non-reentrant tachycardia" in which a patient was found to have 3 distinct AV nodal pathways and multiple "triple fire responses", both on surface ECG and intracardiac electrograms.
\end{abstract}

\section{An Unusual Case of Non-Reentrant Atrioventricular Nodal Tachycardia}

Craig W. Raphael MD

Behzad B. Pavri MD, FACC, FHRS

Thomas Jefferson University Hospital

Philadelphia, PA

Corresponding Author: Behzad Pavri, MD, FACC, FHRS

Email: behzad.pavri@jefferson.edu

Telephone: 215-955-8882

Fax: 215-503-3976

The source data are available for review if needed.

No funding was required for this work.

None of the authors have any conflict of interest pertaining to this work.

This case report is exempt from requirement of IRB approval.

\section{Abstract:}

Although uncommonly encountered, dual atrioventricular nodal non-reentrant tachycardia (DAVNNRT) is a well described arrhythmia that can manifest in patients with dual atrioventricular nodal pathways physiology. This arrhythmia is characterized on ECG by a single $\mathrm{P}$ wave followed by two conducted QRS complexes, and on intracardiac EGMs by a single atrial electrogram followed by two separate His deflections and ventricular electrograms. Our case demonstrates a rare case of "triple atrioventricular non-reentrant tachycardia" in which a patient was found to have 3 distinct AV nodal pathways and multiple "triple fire responses", both on surface ECG and intracardiac electrograms. 


\section{Case Report:}

A 54-year-old man with no previous past medical history presented to his primary care physician with a oneyear history of progressive dyspnea on exertion. On examination, he was found to have resting tachycardia with a heart rate ranging from 150-180 bpm; physical examination was notable for lower extremity edema and pulmonary rales. A transthoracic echocardiogram revealed a severely reduced EF of $20 \%$ with significantly elevated LA pressure. Multiple initial 12-lead ECGs (obtained within hours of each other) demonstrated a narrow complex tachycardia, at times with grouped beating (Figure 1A).

The patient was admitted to the hospital for suspected tachycardia-induced cardiomyopathy and was treated with IV diuresis and initiation of guideline-driven medical therapy for heart failure. Following his admission, telemetry was interpreted as showing periods of "ventricular bigeminy" (Figure 1B) and "ventricular couplets" (Figure 1C). Heart rate trends showed incessant tachycardia (figure 1D).

Electrophysiology consultation recommended ablation, and the patient was brought to the Electrophysiology (EP) laboratory.

\section{Electrophysiology Study Results:}

Bilateral femoral venous access was obtained, and catheters were positioned in the high right atrium, the His bundle region, and the coronary sinus. A mapping/ablating catheter was introduced after the diagnosis was established.

At baseline, intracardiac recordings from the His-bundle region revealed a single atrial electrogram followed by two distinct His deflections (H1 and H2) and two ventricular electrograms (Figure 2A). At a sinus cycle length of $1150 \mathrm{~ms}$, the AH1 and AH2 intervals were consistently 90-100 ms and 435-450 ms, and the HV1 and HV2 intervals were $55 \mathrm{~ms}$ and $65 \mathrm{~ms}$, respectively (see Table). On the surface ECG, the first His signal conducted with a narrow QRS complex (QRS duration $75 \mathrm{~ms}$ ) and the second His signal conducted with either a narrow QRS complex or right-bundle branch block morphology (QRS duration $130 \mathrm{~ms}$ ), due to rate related RBBB aberrancy. Atrial burst pacing at a cycle length of $780 \mathrm{~ms}$ resulted in "double fire, with a ventricular rate of $155 \mathrm{BPM}$ (figure 2B). Atrial burst pacing at a faster cycle length of 450 ms revealed persistent block in the slow pathway, leading to sustained 1:1 AV conduction over the fast pathway and a paradoxical slowing of the ventricular rate to 133 BPM (figure 2C). Ventricular burst pacing demonstrated absence of retrograde conduction.

It was observed on multiple instances that the patient had athird, even wider QRS (140 ms) following some sinus $\mathrm{P}$ waves (figure 3 ), similar to what was observed on telemetry (figure 1C). The intracardiac electrograms on the His bundle catheter demonstrated athird His bundle deflection preceding the $3{ }^{\text {rd }} \mathrm{QRS}$, representing triple AV nodal conduction from the preceding atrial impulse (so-called "triple fire"). Each instance of the 3rd response conducted with markedly prolonged AH interval of $730 \mathrm{~ms}$; each "triple fire" was followed by a sinus $\mathrm{P}$ waves with a single QRS response.

Table: The electrophysiologic characteristics associated with the $3 \mathrm{AV}$ nodal pathways are summarized in the Table:

\begin{tabular}{llll}
\hline & PR (ms) & AH (ms) & HV (ms) \\
\hline Fast Pathway & $170-190$ & $90-100$ & 55 \\
Slow Pathway & $530-540$ & $435-450$ & $65-75$ \\
Slowest Pathway & 840 & 730 & 85 \\
\hline
\end{tabular}

(The PA interval was 25-30 ms)

Ablation

Once the arrhythmia mechanism was definitively established, radiofrequency (RF) ablation of the putative 
slow pathway was performed. An externally irrigated, contact force sensing catheter was positioned via a deflectable sheath in the posterior interatrial septum. At sites that showed low-amplitude, multiphasic atrial signals and large ventricular signals, a total of $8 \mathrm{RF}$ energy applications were delivered using 30 watts of power with low flow rate of $2 \mathrm{ml}$; contact force was kept between 8 and 15 grams; duration of application ranged between 10 and 20 seconds. Development of junctional rhythm without retrograde conduction was noted, and would prompt termination of RF delivery.

Following the $5^{\text {th }} \mathrm{RF}$ application, repetitive fire terminated, and normal sinus rhythm with 1:1 AV conduction was seen. Repeat atrial and ventricular programmed stimulation (without and with intravenous isoproterenol infusion) failed to reveal dual AVN physiology or reinitiate the tachycardia. At outpatient follow up visit several weeks later, he reported complete resolution of heart failure symptoms, an ECG showed normal sinus rhythm.

\section{Discussion}

Dual atrioventricular nodal pathways physiology refers to the functional dissociation of conduction through the atrioventricular node wherein atrial impulses conduct with two distinct PR intervals (2 "families" of PR interval). Myriad clinical and ECG presentations are known to result from dual pathways physiology. ${ }^{1}$ Most commonly, dual pathways physiology can manifest as typical atrioventricular nodal reentrant tachycardia (AVNRT), a form of reentrant tachycardia with antegrade conduction down the "slow pathway" and retrograde conduction up the "fast pathway" of the AV node.

An uncommon presentation of dual AV nodal pathways physiology is "dual atrioventricular nodal nonreentrant tachycardia" (DAVNNRT), in which a single atrial impulse conducts antegradely down both AV nodal pathways resulting in two separate ventricular depolarizations, also called "double fire tachycardia". For this to occur, several conditions must be met: 1) A large difference in conduction velocities of each pathway; 2) absent/poor retrograde fast pathway conduction; and 3) refractory period of the distal common final pathway is short enough to allow conduction of the second wave front from the slow pathway. ${ }^{2}$ There are numerous case reports of such patients presenting with heart failure due to tachycardia-related cardiomyopathy, which is typically reversible.

Numerous reports have also been published of multiple antegrade "jumps" (AH increment of $>50 \mathrm{~ms}$ with $10 \mathrm{~ms}$ decrement in atrial coupling interval), which have been interpreted as being reflective of multiple AV nodal pathways. ${ }^{3-5}$ However, more than a dual antegrade conduction has only rarely been described. ${ }^{6-9}$

Our case demonstrates a most unusual presentation: "Triple-fire tachycardia" due to triple antegrade AV nodal pathways physiology, with the longest PR interval of $840 \mathrm{~ms}$. This finding was repeatedly demonstrated throughout the study. The marked difference in conduction velocities of the second and third AVN pathways (AH2 versus AH3 $>250 \mathrm{~ms}$ ) is consistent with the criteria to allow for multiple, separate ventricular depolarizations. The documentation of a $3^{\text {rd }}$ QRS complex on telemetry excludes catheter-induced His extra-systoles. As is usually the case, ablation in the posterior interatrial septum abolished repetitive firing over all but the "fast pathway". This case highlights the remarkable variations in human AV nodal physiology, remarkably slow antegrade conduction in the absence of retrograde conduction, and the potential to cause cardiac pathology.

\section{References:}

1. Mani BC, Pavri BB. Dual atrioventricular nodal pathways physiology: A review of relevant anatomy, electrophysiology, and electrocardiographic manifestations. Indian Pacing Electrophysiol J . Published online 2014. doi:10.1016/S0972-6292(16)30711-2

2. Wang NC. Dual Atrioventricular nodal nonreentrant tachycardia: A systematic review. PACE - Pacing Clin Electrophysiol . Published online 2011. doi:10.1111/j.1540-8159.2011.03218.x

3. Centurion OA, Isomoto S, Hayano M, Yano K. Evidence of quadruple anterograde atrioventricular nodal pathways in a patient with atrioventricular node reentry. J Electrocardiol . Published online 1994. 
doi:10.1016/S0022-0736(05)80112-8

4. Dopirak MR, Schaal SF, Leier C V. Triple AV nodal pathways in man? J Electrocardiol . Published online 1980. doi:10.1016/S0022-0736(80)80052-5

5. Swiryn S, Bauernfeind RA, Palileo EA, Strasberg B, Duffy CE, Rosen KM. Electrophysiologic study demonstrating triple antegrade AV nodal pathways in patients with spontaneous and/or induced supraventricular tachycardia. Am Heart $J$. Published online 1982. doi:10.1016/0002-8703(82)90489-6

6. Lee KL, Chun HM, Liem LB, Lauer MR, Young C, Sung RJ. Multiple atrioventricular nodal pathways in humans: Electrophysiologic demonstration and characterization. J Cardiovasc Electrophysiol . Published online 1998. doi:10.1111/j.1540-8167.1998.tb00894.x

7. Peng D, Liu X, Pan Y. Irregular Complex Tachycardia: Not Dual Atrioventricular Nodal Nonreentrant Tachycardia. Circulation . Published online 2021. doi:10.1161/CIRCULATIONAHA.120.052913

8. Arena G, Bongiorni MG, Soldati E, Gherarducci G, Mariani M. Incessant nonreentrant atrioventricular nodal tachycardia due to multiple nodal pathways treated by radiofrequency ablation of the slow pathways. $J$ Cardiovasc Electrophysiol . Published online 1999. doi:10.1111/j.1540-8167.1999.tb00228.x

9. Yokoshiki H, Sasaki K, Shimokawa J, Sakurai M, Tsutsui H. Nonreentrant atrioventricular nodal tachycardia due to triple nodal pathways manifested by radiofrequency ablation at coronary sinus ostium. $J$ Electrocardiol . Published online 2006. doi:10.1016/j.jelectrocard.2005.08.017

\section{Hosted file}

Triple Fire Case Report Figures and Figure Legends.docx available at https://authorea.com/ users/453728/articles/551481-an-unusual-case-of-non-reentrant-atrioventricular-nodaltachycardia 